\title{
Existence and multiplicity of solutions for a second-order impulsive differential equation via variational methods
}

\author{
Yaqi Wang ${ }^{1 *}$ and Zengqin Zhao ${ }^{2}$
}

"Correspondence:

yqwtengzhou@126.com

'Southeast University, Nanjing, P.R.

China

Full list of author information is

available at the end of the article

\begin{abstract}
In this paper, we study the existence and multiplicity of solutions for an impulsive differential equation via some critical point theory and the variational method. We extend and improve some recent results and reduce conditions.
\end{abstract}

Keywords: impulsive differential equation; critical point theory; variational structure

\section{Introduction}

As an important research field of study, the impulsive differential equation has been attracting the attention of several mathematicians. In the early years, the main way to resolve this kind of problems is based on the fixed point theory, the theorem of topological degree, the upper and lower solutions method coupled with the monotone iterative technique, and so on; see for example [1-3]. Recently, many authors have tried to use the variational method and some specific critical point theorems, such as mountain pass lemma, fountain theorem, linking theorem, symmetric mountain pass lemma, and so on, to study the existence (see [4-9]) and multiplicity (see [10-15]) of solutions for some impulsive differential equations.

In [5], authors have shown the variational structure of an impulsive differential equation and proved the existence of a solution by using the mountain pass lemma.

In [10], the authors studied the following equation:

$$
\begin{cases}-\left(p(t) u^{\prime}\right)^{\prime}(t)+r(t) u^{\prime}(t)+q(t) u(t)=f(t, u(t)), & \text { a.e. } t \in J, \\ -\triangle\left(p(t) u^{\prime}\left(t_{i}\right)\right)=I_{i}\left(u\left(t_{i}\right)\right), & i=1,2, \ldots, p, \\ u(0)=0, \quad \alpha_{1} u(t)+u^{\prime}(1)=0, & \end{cases}
$$

where $\left.J=[0,1], 0=t_{0}<t_{1}<t_{2}<\cdots<t_{p}<t_{p+1}=1, f \in C U \times R, R\right], p \in C\left[J, R^{+}\right], q, r \in$ $C[0, T]$.

\section{Springer}


Then Zhang in [11] proved the existence of two solutions and the existence of infinitely many solutions of problem given by

$$
\begin{cases}-u^{\prime \prime}(t)+\lambda u(t)=f(t, u(t)), & \text { a.e. } t \in[0, T], \\ \triangle u^{\prime}\left(t_{i}\right)=I_{i}\left(u\left(t_{i}\right)\right), & i=1,2, \ldots, p \\ u(0)=u(T)=0 . & \end{cases}
$$

In this paper, we study the existence and multiplicity of solutions for the following nonlinear impulsive problem:

$$
\begin{cases}-u^{\prime \prime}(t)+r(t) u^{\prime}(t)+\lambda u(t)=f(t, u(t)), & \text { a.e. } t \in J, \\ \triangle u^{\prime}\left(t_{i}\right)=u^{\prime}\left(t_{i}+\right)-u^{\prime}\left(t_{i}-\right)=I_{i}\left(u\left(t_{i}\right)\right), & i=1,2, \ldots, p, \\ u(0)=u(T)=0, & \end{cases}
$$

where $J=[0, T], 0=t_{0}<t_{1}<t_{2}<\cdots<t_{p}<t_{p+1}=T, r \in C[0, T], I_{i} \in C[R, R], \lambda$ is a parameter, $f \in C[J \times R, R]$, with $F(t, u)=\int_{0}^{u} f(t, \xi) d \xi$.

We will prove that equation (1) has at least two classical solutions and infinitely many classical solutions under different conditions. Our main results extend the existing result in $[5,10,11]$. We prove the same impulsive problem in [5] cannot only have two solutions but also have infinitely many classical solutions. Compared with [10], we do not require the impulsive functions $I_{j}$ and $F$ to satisfy the sublinear growth condition and the superlinear growth condition about $u^{\mu}$, which such that the problem more general. Different from [11] in which $F$ is a negative function, in this paper, our results relax the restriction of $F$ with a wider range of applications.

The rest of the paper is organized as follows: In Section 2, we give the variational structure and several important lemmas. The main theorems are formulated and proved in Section 3.

\section{Preliminaries}

Let $R(t)=\int_{0}^{t} r(s) d s, M=\max _{t \in[0, T]} e^{-R(t)}, m=\min _{t \in[0, T]} e^{-R(t)}$. Multiplying the first equation of (1) by $e^{-R(t)}$, we obtain

$$
\begin{cases}-\left(e^{-R(t)} u^{\prime}(t)\right)^{\prime}+\lambda e^{-R(t)} u(t)=e^{-R(t)} f(t, u(t)), & \text { a.e. } t \in J, \\ \triangle u^{\prime}\left(t_{i}\right)=I_{i}\left(u\left(t_{i}\right)\right), & i=1,2, \ldots, p, \\ u(0)=u(T)=0 . & \end{cases}
$$

Obviously, the solutions of equation (2) are solutions of equation (1). Consider the Hilbert space $H_{0}^{1}(0, T)$ with the inner product and norm

$$
\begin{aligned}
& (u, v)=\int_{0}^{T} e^{-R(t)} u^{\prime}(t) v^{\prime}(t) d t, \\
& \|u\|=\left(\int_{0}^{T} e^{-R(t)}\left(u^{\prime}(t)\right)^{2} d t\right)^{1 / 2} .
\end{aligned}
$$


Multiply the first equation of (2) by $v \in H_{0}^{1}(0, T)$, integrate it on the interval $[0, T]$. It follows from the boundary conditions $u(0)=0, u(T)=0$ that

$$
\begin{aligned}
& \int_{0}^{T} e^{-R(t)} u^{\prime}(t) v^{\prime}(t) d t+\lambda \int_{0}^{T} e^{-R(t)} u(t) v(t) d t+\sum_{i=1}^{p} e^{-R(t)} I_{i}\left(u\left(t_{i}\right)\right) v\left(t_{i}\right) \\
& \quad=\int_{0}^{T} e^{-R(t)} f(t, u(t)) v(t) d t .
\end{aligned}
$$

Now, define the function $\varphi: H_{0}^{1}(0, T) \rightarrow R$

$$
\begin{aligned}
\varphi(u)= & L(u, u)+\sum_{i=1}^{p} e^{-R(t)} \int_{0}^{u\left(t_{i}\right)} I_{i}(s) d s \\
& -\int_{0}^{T} e^{-R(t)} F(t, u(t)) d t
\end{aligned}
$$

where

$$
L(u, v)=\frac{1}{2} \int_{0}^{T} e^{-R(t)} u^{\prime}(t) v^{\prime}(t) d t+\frac{\lambda}{2} \int_{0}^{T} e^{-R(t)} u(t) v(t) d t
$$

and for all $v \in H_{0}^{1}(0, T)$, we have

$$
\begin{aligned}
\varphi^{\prime}(u) v= & \int_{0}^{T} e^{-R(t)} u^{\prime}(t) v^{\prime}(t) d t+\lambda \int_{0}^{T} e^{-R(t)} u(t) v(t) d t \\
& +\sum_{i=1}^{p} e^{-R(t)} I_{i}\left(u\left(t_{i}\right)\right) v\left(t_{i}\right)-\int_{0}^{T} e^{-R(t)} f(t, u(t)) v(t) d t .
\end{aligned}
$$

Definition 1 A weak solution of problem (1) is a function $u \in H_{0}^{1}(0, T)$ such that the integral equation (3) holds for all $v \in H_{0}^{1}(0, T)$.

Let $\lambda_{k}(k=1,2, \ldots)$ be the eigenvalue of the following Dirichlet problem, where $\lambda_{1}=\frac{\pi^{2}}{T^{2}}$ is the first eigenvalue:

$$
\left\{\begin{array}{l}
-u^{\prime \prime}(t)=\lambda u(t), \quad t \in[0, T] \\
u(0)=u(T)=0
\end{array}\right.
$$

We assume $X_{k}$ is the feature space corresponding with $\lambda_{k}$, then $H_{0}^{1}(0, T)=\overline{\bigoplus_{i \in N} X_{i}}$.

Lemma 1 There exists a constant $C>0$, such that $\|u\|_{\infty} \leq C\|u\|$, where $\|u\|_{\infty}=$ $\max _{t \in[0, T]}|u(t)|$.

Lemma 2 ([5]) If $\lambda>-\frac{m \lambda_{1}}{M}$, then there exist $0<a_{1}<a_{2}$, such that

$$
a_{1}\|u\|^{2} \leq L(u, u) \leq a_{2}\|u\|^{2}
$$


Remark 1 If $|\lambda|<\frac{m \lambda_{1}}{M}$, then $0<a_{1}<a_{2}<1$. In fact, if $\lambda>0$, then let $a_{1}=\frac{1}{2}$, and by the Poincaré inequality we have

$$
\begin{aligned}
L(u, u) & =\frac{1}{2} \int_{0}^{T} e^{-R(t)}\left(u^{\prime}(t)\right)^{2} d t+\frac{\lambda}{2} \int_{0}^{T} e^{-R(t)} u^{2}(t) d t \\
& \leq \frac{1}{2}\|u\|^{2}+\frac{\lambda M}{2} \int_{0}^{T} u^{2}(t) d t \\
& \leq \frac{1}{2}\|u\|^{2}+\frac{\lambda M}{2 \lambda_{1}} \int_{0}^{T}\left(u^{\prime}(t)\right)^{2} d t \\
& \leq \frac{1}{2}\|u\|^{2}+\frac{\lambda M}{2 m \lambda_{1}} \int_{0}^{T} e^{-R(t)}\left(u^{\prime}(t)\right)^{2} d t \\
& =\left(\frac{1}{2}+\frac{\lambda M}{2 m \lambda_{1}}\right)\|u\|^{2} .
\end{aligned}
$$

Then letting $a_{2}=\frac{1}{2}+\frac{\lambda M}{2 m \lambda_{1}}$, we can get the result. Similarly, if $-\frac{m \lambda_{1}}{M}<\lambda<0$, then let $a_{2}=\frac{1}{2}$, and by the Poincaré inequality we have

$$
\begin{aligned}
L(u, u) & =\frac{1}{2} \int_{0}^{T} e^{-R(t)}\left(u^{\prime}(t)\right)^{2} d t+\frac{\lambda}{2} \int_{0}^{T} e^{-R(t)} u^{2}(t) d t \\
& \geq \frac{1}{2}\|u\|^{2}+\frac{\lambda M}{2} \int_{0}^{T} u^{2}(t) d t \\
& \geq \frac{1}{2}\|u\|^{2}+\frac{\lambda M}{2 \lambda_{1}} \int_{0}^{T}\left(u^{\prime}(t)\right)^{2} d t \\
& \geq \frac{1}{2}\|u\|^{2}+\frac{\lambda M}{2 m \lambda_{1}} \int_{0}^{T} e^{-R(t)}\left(u^{\prime}(t)\right)^{2} d t \\
& =\left(\frac{1}{2}+\frac{\lambda M}{2 m \lambda_{1}}\right)\|u\|^{2} .
\end{aligned}
$$

We can also let $a_{1}=\frac{1}{2}+\frac{\lambda M}{2 m \lambda_{1}}$ to get the result.

Lemma 3 ([16]) For the function $F: M \subseteq E \rightarrow R$ with $M \neq \emptyset, \min _{u \in M} F(u)=\alpha$ has a solution in the case that the following hold:

$\left(F_{1}\right) E$ is a real reflexive Banach space,

$\left(F_{2}\right) M$ is bounded and weak sequentially closed,

$\left(F_{3}\right) F$ is weak sequentially lower semi-continuous on $M$, i.e., by definition, for each sequence $\left\{u_{n}\right\}$ in $M$ such that $u_{n} \rightarrow u$ as $n \rightarrow \infty$, we have $F(u) \leq \liminf _{n \rightarrow \infty} F\left(u_{n}\right)$.

Lemma $4([6])$ Let $E$ be a real Banach space with $E=V \bigoplus W$, where $V$ is finitedimensional. Suppose $\phi \in C^{\prime}(E, R)$ satisfies P.S. condition, and:

$\left(\phi_{1}\right)$ there are constants $\rho, \tau>0$ such that $\left.\phi\right|_{\partial B_{\rho} \cap W} \geq \tau$, and

$\left(\phi_{2}\right)$ there is $e \in \partial B_{1} \cap W$ and $R>\rho$ such that if $Q=\left(\overline{B_{R}} \cap V\right) \oplus\{r e \mid 0<r<R\}$, then $\left.\phi\right|_{\partial Q} \leq 0$.

Then $\phi$ possesses a critical value $c \geq \tau$ which can be characterized as $c=\inf _{h \in \Gamma} \max _{u \in Q} \phi(h(u))$, where $\Gamma=\left\{h \in C(\bar{Q}, E),\left.h\right|_{\partial Q}=i d\right\}$. 
Lemma 5 ([17]) Let $E$ be an infinite-dimensional real Banach space and $\varphi \in C^{1}(E, R)$ be even, satisfying the P.S. condition and $u(0)=0$. If $E=V \oplus W$, where $V$ is finite-dimensional, and $\varphi$ satisfies the following conditions:

$\left(\varphi_{1}\right)$ there exist constants $\rho, \tau>0$, such that $\left.\varphi\right|_{\partial B_{\rho} \cap W} \geq \tau$,

$\left(\varphi_{2}\right)$ for each finite-dimensional subspace $V_{1} \subset E$, there is $R=R\left(V_{1}\right)$ such that, for all $u \in\left\{u \in V_{1},\|u\| \geq R\right\}$, we have $\varphi(u) \leq 0$.

Then $\varphi$ has an unbounded sequence of critical values.

\section{Main results}

In this paper, we assume:

$\left(H_{1}\right)$ There exists $\mu>2$ such that $\mu F(t, u) \leq u f(t, u)$ and $I_{i}(u) u \leq \mu \int_{0}^{u} I_{i}(s) d s<0$.

$\left(H_{2}\right)$ There exists $\rho_{0}>0, \delta_{i}>0$ such that $\int_{0}^{u} I_{i}(s) d s \geq-\delta_{i}|u|^{\mu}$ for all $\|u\| \leq \rho_{0}$.

$\left(H_{3}\right)$ For all $u \in X_{1} \cup X_{2}$, we have $\int_{0}^{T} F(t, u(t)) d t \geq \frac{a_{2}\|u\|^{2}}{m}$.

The main results are the following theorems.

Theorem 1 Assume $\left(H_{1}\right)-\left(H_{3}\right)$ hold and $|\lambda|<\frac{m \lambda_{1}}{M}$, then the impulsive problem (1) has at least two weak solutions.

Theorem 2 Suppose that $\left(H_{1}\right)-\left(H_{2}\right)$ are satisfied and $\lambda \geq-\frac{m \lambda_{1}}{M}$. Iff $(t, u)$ and $I_{i}(u)$ are odd about $u$, then the problem (1) has infinitely many weak solutions.

Next, we give the main lemma used in this paper.

Lemma 6 ([12]) Suppose that $\left(H_{1}\right)$ holds, then for all $t \in[0, T]$ we can obtain

$$
\begin{aligned}
& F(t, u) \leq F\left(t, \frac{u}{|u|}\right)|u|^{\mu}, \quad 0<|u| \leq 1, \\
& F(t, u) \geq F\left(t, \frac{u}{|u|}\right)|u|^{\mu}, \quad|u| \geq 1 .
\end{aligned}
$$

Remark 2 For the convenience of the reader, we denote $M_{1}=\sup \{F(t, u), t \in[0, T],|u|=$ $1\}, m_{1}=\inf \{F(t, u), t \in[0, T],|u|=1\}$.

Lemma 7 Suppose that $\left(H_{1}\right)$ and $\left(H_{2}\right)$ hold, then $\varphi$ satisfies the P.S. condition.

Proof Let $\left\{u_{n}\right\} \in H_{0}^{1}(0, T)$ be such a sequence that $\left\{\varphi\left(u_{n}\right)\right\}$ is bounded and $\lim _{n \rightarrow \infty} \varphi^{\prime}\left(u_{n}\right)=$ 0 , we will show that $u_{n}$ has a convergent subsequence. In view of the given condition, there exists $C_{1}>0$ such that

$$
\left|\varphi\left(u_{n}\right)\right| \leq C_{1}, \quad\left\|\varphi^{\prime}\left(u_{n}\right)\right\| \leq C_{1}
$$

for all $n$. By the definition of $\varphi$ and (3) we obtain

$$
\begin{aligned}
& \left(1-\frac{2}{\mu}\right)\left(\int_{0}^{T} e^{-R(t)}\left(u^{\prime}(t)\right)^{2} d t+\int_{0}^{T} e^{-R(t)} u^{2}(t) d t\right) \\
& =2 \varphi(u)-2 \sum_{i=1}^{p} e^{-R(t)} \int_{0}^{u_{n}\left(t_{i}\right)} I_{i}(s) d s+2 \int_{0}^{T} e^{-R(t)} F\left(t, u_{n}(t)\right) d t
\end{aligned}
$$




$$
\begin{aligned}
& -\frac{2}{\mu} \varphi^{\prime}\left(u_{n}\right) u_{n}+\frac{2}{\mu} \sum_{i=1}^{p} e^{-R(t)} I_{i}\left(u_{n}\left(t_{i}\right)\right) u_{n}-\frac{2}{\mu} \int_{0}^{T} e^{-R(t)} f\left(t, u_{n}(t)\right) u_{n}(t) d t \\
= & 2 C_{1}+\frac{2}{\mu} C_{1}\left\|u_{n}\right\|+2 \sum_{i=1}^{p} e^{-R(t)}\left[\frac{1}{\mu} I_{i}\left(u_{n}\left(t_{i}\right)\right) u_{n}-\int_{0}^{u_{n}\left(t_{i}\right)} I_{i}(s) d s\right] \\
& +2 \int_{0}^{T} e^{-R(t)}\left[F\left(t, u_{n}(t)\right)-\frac{1}{\mu} f\left(t, u_{n}(t)\right) u_{n}(t)\right] d t .
\end{aligned}
$$

Combining $\left(H_{1}\right)$, we have

$$
\left(1-\frac{2}{\mu}\right) 2 a_{1}\left\|u_{n}\right\|^{2} \leq 2 C_{1}+\frac{2}{\mu} C_{1}\left\|u_{n}\right\|
$$

This implies that $\left\{u_{n}\right\}$ is bounded and, if necessary going to a sequence we can suppose that $u_{n} \rightarrow u \in H_{0}^{1}(0, T)$. By (3) and Lemma 2 we have

$$
\begin{aligned}
\left(\varphi^{\prime}\left(u_{n}\right)-\varphi^{\prime}(u)\right)\left(u_{n}-u\right)= & \int_{0}^{T} e^{-R(t)}\left(u_{n}^{\prime}(t)-u^{\prime}(t)\right)^{2} d t+\lambda \int_{0}^{T} e^{-R(t)}\left(u_{n}-u_{n}(t)\right)^{2} d t \\
& +\sum_{i=1}^{p} e^{-R(t)}\left[I_{i}\left(u_{n}\left(t_{i}\right)\right)-I_{i}\left(u\left(t_{i}\right)\right)\right]\left(u_{n}(t)-u(t)\right) \\
& -\int_{0}^{T} e^{-R(t)}\left[f\left(t, u_{n}(t)\right)-f(t, u(t))\right]\left(u_{n}(t)-u(t)\right) d t \\
\geq & 2 a_{1}\left\|u_{n}-u\right\|^{2}+\sum_{i=1}^{p} e^{-R(t)}\left[I_{i}\left(u_{n}\left(t_{i}\right)\right)-I_{i}\left(u\left(t_{i}\right)\right)\right]\left(u_{n}(t)-u(t)\right) \\
& -\int_{0}^{T} e^{-R(t)}\left[f\left(t, u_{n}(t)\right)-f(t, u(t))\right]\left(u_{n}(t)-u(t)\right) d t .
\end{aligned}
$$

Hence, $u_{n} \rightarrow u$ in $C[0, T]$. Furthermore

$$
\begin{aligned}
& \left(\varphi^{\prime}\left(u_{n}\right)-\varphi^{\prime}(u)\right)\left(u_{n}-u\right) \rightarrow 0, \\
& \sum_{i=1}^{p} e^{-R(t)}\left[I_{i}\left(u_{n}\left(t_{i}\right)\right)-I_{i}\left(u\left(t_{i}\right)\right)\right]\left(u_{n}(t)-u(t)\right) \rightarrow 0, \\
& \int_{0}^{T} e^{-R(t)}\left[f\left(t, u_{n}(t)\right)-f(t, u(t))\right]\left(u_{n}(t)-u(t)\right) d t \rightarrow 0 .
\end{aligned}
$$

Combining with (6), we know $\left\|u_{n}-u\right\|^{2} \rightarrow 0$. So, $\varphi$ satisfies the P.S. condition.

Proof of Theorem 1 Because $H_{0}^{1}(0, T)$ is Hilbert space, $\overline{B_{\rho}}$ is bounded and weak sequentially closed for all $\rho>0$. We will show $\varphi$ is weak sequentially lower semi-continuous on $\overline{B_{\rho}}$. In fact suppose $u_{n} \rightarrow u$ in $H_{0}^{1}(0, T)$, then $\|u\| \leq \liminf _{n \rightarrow \infty}\left\|u_{n}\right\|$, and $u_{n} \rightarrow u$ in $C[0, T]$, so

$$
\begin{aligned}
& \frac{\lambda}{2} \int_{0}^{T} e^{-R(t)} u_{n}^{2}(t) d t+\sum_{i=1}^{p} e^{-R(t)} \int_{0}^{u_{n}\left(t_{i}\right)} I_{i}(s) d s-\int_{0}^{T} e^{-R(t)} F\left(t, u_{n}(t)\right) d t \\
& \rightarrow \frac{\lambda}{2} \int_{0}^{T} e^{-R(t)} u^{2}(t) d t+\sum_{i=1}^{p} e^{-R(t)} \int_{0}^{u\left(t_{i}\right)} I_{i}(s) d s-\int_{0}^{T} e^{-R(t)} F(t, u(t)) d t .
\end{aligned}
$$


By the definition of $\varphi$, we can obtain $\varphi(u) \leq \liminf _{n \rightarrow \infty} \varphi\left(u_{n}\right)$. From Lemma 3, there exists $u_{0}$ such that $\varphi\left(u_{0}\right)=\min \left\{\varphi(u), u \in \overline{B_{\rho}}\right\}$. Noting that $\varphi(0)=0$, so $\varphi\left(u_{0}\right) \leq 0$. Combining with Lemma 2, Lemma 6 , and $\left(H_{2}\right)$, there exists a small $\rho$ with $\rho<\rho_{0}$ such that when $u(t) \in \partial B_{\rho}$ we have

$$
\begin{aligned}
\varphi(u) & \geq a_{1}\|u\|^{2}+\sum_{i=1}^{p} e^{-R(t)} \int_{0}^{u\left(t_{i}\right)} I_{i}(s) d s-\int_{0}^{T} e^{-R(t)} F(t, u(t)) d t \\
& \geq a_{1}\|u\|^{2}-\sum_{i=1}^{p} e^{-R(t)} \delta_{i}|u|^{\mu}-m_{1} \int_{0}^{T} e^{-R(t)}|u(t)|^{\mu} d t \\
& \geq a_{1}\|u\|^{2}-\left(\sum_{i=1}^{p} \delta_{i} M+\delta M m_{1} T\right) C\|u\|^{\mu} \\
& =a_{1} \rho^{2}-\left(\sum_{i=1}^{p} \delta_{i} M+\delta M m_{1} T\right) C \rho^{\mu}=\tau>0 .
\end{aligned}
$$

$\varphi\left(u_{0}\right) \leq 0$ implies that $\varphi$ possesses a critical point $u_{0} \in B_{\rho}$.

Let $V=X_{1} \oplus X_{2}, W=\overline{\bigoplus_{i=3}^{\infty} X_{i}}$, then there exists $\rho, \tau>0$, such that

$$
\left.\varphi\right|_{\partial B_{\rho} \cap W} \geq \tau
$$

Hence $\varphi$ satisfies the condition $\left(\phi_{1}\right)$.

Taking $e \in W$ such that $\|e\|=1$. By Lemma 6 , we claim that there exist $C_{2}, M_{2}>0$, such that

$$
\begin{aligned}
& \|e\| \leq C_{2}|e|_{L^{2}}, \quad\|u\| \leq C_{2}|u|_{L^{2}}, \quad u \in V, \\
& F(t, u) \geq C_{2}^{2}|u|^{2}-M_{2}, \quad(t, u) \in[0, T] \times R .
\end{aligned}
$$

By $\left(H_{1}\right)$, for all $r>0, u \in V$, we have

$$
\begin{aligned}
\varphi(r e+u) & =L(r e+u, r e+u)+\sum_{i=1}^{p} e^{-R(t)} \int_{0}^{r e+u} I_{i}(s) d s-\int_{0}^{T} e^{-R(t)} F(t, r e+u) d t \\
& \leq a_{2}\|r e+u\|^{2}-\int_{0}^{T} e^{-R(t)}\left(C_{2}^{2}|r e+u|^{2}-M_{2}\right) d t \\
& \leq a_{2} r^{2}+a_{2}\|u\|^{2}+2 a_{2} r\|u\|+T M_{2} M-C_{2}^{2}\left(|r e|_{L^{2}}^{2}+|u|_{L^{2}}^{2}-2|r e|_{L^{2}}|u|_{L^{2}}\right) \\
& \leq a_{2} r^{2}+a_{2}\|u\|^{2}+2 a_{2} r\|u\|+T M_{2} M-r^{2}-\|u\|^{2}+2 r\|u\| \\
& =-\left(1-a_{2}\right) r^{2}-\left(1-a_{2}\right)\|u\|^{2}+2\left(a_{2}+1\right) r\|u\|+T M_{2} M .
\end{aligned}
$$

Combining with $a_{2}<1$, there exists $R>0$, such that the following conclusions hold:

when $\|u\| \geq R, \varphi(r e+u)<0 \quad$ for all $0<r<R$;

when $r \geq R, \varphi(r e+u)<0 \quad$ for all $\|u\| \leq R$. 
For any $u(t) \in V,\|u\| \leq R$, we can obtain

$$
\varphi(u) \leq a_{2}\|u\|^{2}-m \int_{0}^{T} F(t, u(t)) d t \leq 0 .
$$

Let $\Omega=\left(\overline{B_{R}} \cap V\right) \oplus\{r e \mid 0<r<R\}, \partial \Omega=\Omega_{1} \cup \Omega_{2} \cup \Omega_{3}$, where

$$
\begin{aligned}
& \Omega_{1}=\{u \in V,\|u\| \leq R\}, \quad \Omega_{2}=R e \oplus\{u \in V,\|u\| \leq R\}, \\
& \Omega_{3}=\{r e \mid 0<r<R\} \oplus\{u \in V,\|u\|=R\} .
\end{aligned}
$$

So $\left.\varphi\right|_{\partial} Q \leq 0$. From Lemma 4 we know that $\varphi$ has a critical point $c$ with $c \geq \tau>0$. In other words, there exists $u_{1}$ such that $\varphi\left(u_{1}\right)=c$. Hence $u_{0}$ and $u_{1}$ are classical solutions of the impulsive problem (1).

Proof of Theorem 2 Since $f(t, u)$ and $I_{i}$ are odd about $u, \varphi(u)$ is even and $\varphi(0)=0$. Lemma 7 shows that $\varphi$ satisfies the P.S. condition. In the same way as in Theorem 1 , we can verify $\varphi$ satisfies the condition $\left(\varphi_{1}\right)$ in Lemma 5. Finally we prove that $\varphi$ also satisfies the condition $\left(\varphi_{2}\right)$. According to Lemma 6 and $\left(H_{1}\right)$, for every $V_{1}, u \in V_{1}$, there exists $C_{3}, M_{3}>0$ such that

$$
\begin{aligned}
\varphi(u) & \leq a_{2}\|u\|^{2}+\sum_{i=1}^{p} e^{-R(t)} \int_{0}^{u\left(t_{i}\right)} I_{i}(s) d s-\int_{0}^{T} e^{-R(t)} F(t, u(t)) d t \\
& \leq a_{2}\|u\|^{2}-m \int_{0}^{T}\left(m_{1}|u(t)|^{\mu}-C_{3}\right) d t \\
& \leq a_{2}\|u\|^{2}-M_{3}\|u\|^{\mu}+m T C_{3},
\end{aligned}
$$

then we can find $R>0$ such that $\varphi(u) \leq 0$ when $\|u\| \geq R$. By Lemma 5, $\varphi$ possesses infinitely many critical points. Hence, BVP (1) has infinitely many classical solutions.

Example Take $x(t) \in C[0, T], t_{1} \in(0, T)$ and $k>0$. Consider the following impulsive problem:

$$
\left\{\begin{array}{l}
-u^{\prime \prime}(t)+t u^{\prime}(t)+\lambda u(t)=e^{-t} x(t) u^{\frac{5}{3}}(t), \quad t \neq t_{1}, \\
\triangle u^{\prime}\left(t_{1}\right)=u^{\prime}\left(t_{1}+\right)-u^{\prime}\left(t_{1}-\right)=-k u\left(t_{1}\right), \\
u(0)=u(T)=0 .
\end{array}\right.
$$

Let $\mu=2.1, \delta_{1}=1$ and $\rho_{0}=1$, then the differential equation (7) has infinity many weak solutions by Theorem 2 .

\section{Conclusion}

A second-order impulsive differential equation is considered in this paper. Some necessary conditions for the existence and multiplicity of solutions are presented by critical point theories and variational methods. We also proposed a numerical example to show the advantage. 
Competing interests

The authors declare that they have no competing interests.

\section{Authors' contributions}

All authors have made equal contributions. All authors read and approved the final manuscript.

\section{Author details}

${ }^{1}$ Southeast University, Nanjing, P.R. China. ${ }^{2}$ Qufu Normal University, Qufu, P.R. China.

\section{Acknowledgements}

The authors are very grateful to the anonymous referee for his/her valuable comments and suggestions.

Received: 4 November 2016 Accepted: 4 January 2017 Published online: 08 February 2017

\section{References}

1. Lin, Z, Wang, J, Wei, W: Multipoint bvps for generalized impulsive fractional differential equations. Appl. Math. Comput. 258, 608-616 (2015)

2. Fec, M, Zhou, Y, Wang, J, et al.: On the concept and existence of solution for impulsive fractional differential equations. Commun. Nonlinear Sci. Numer. Simul. 17(7), 3050-3060 (2012)

3. Pierri, $M, O$ 'Regan, D, Rolnik, V: Existence of solutions for semi-linear abstract differential equations with not instantaneous impulses. Appl. Math. Comput. 219(12), 6743-6749 (2013)

4. Nieto, JJ: Variational formulation of a damped Dirichlet impulsive problem. Appl. Math. Lett. 23(8), 940-942 (2010)

5. Xiao, J, Nieto, JJ: Variational approach to some damped Dirichlet nonlinear impulsive differential equations. J. Franklin Inst. 348(2), 369-377 (2011)

6. He, X, Wu, X: Periodic solutions for a class of nonautonomous second order Hamiltonian systems. J. Math. Anal. Appl. $341(2), 1354-1364(2008)$

7. Zhang, Z, Yuan, R: An application of variational methods to Dirichlet boundary value problem with impulses. Nonlinear Anal., Real World Appl. 11(1), 155-162 (2010)

8. Nieto, JJ, O'Regan, D: Variational approach to impulsive differential equations. Nonlinear Anal., Real World Appl. 10(2), 680-690 (2009)

9. Luo, Z, Xie, J, Chen, G: Existence of solutions of a second-order impulsive differential equation. Adv. Differ. Equ. 2014(1), $118(2014)$

10. Xiao, J, Nieto, JJ, Luo, Z: Multiplicity of solutions for nonlinear second order impulsive differential equations with linear derivative dependence via variational methods. Commun. Nonlinear Sci. Numer. Simul. 17(1), 426-432 (2012)

11. Zhang, D: Multiple solutions of nonlinear impulsive differential equations with Dirichlet boundary conditions via variational method. Results Math. 63(1-2), 611-628 (2013)

12. Zhou, J, Li, Y: Existence and multiplicity of solutions for some Dirichlet problems with impulsive effects. Nonlinear Anal. 71(7), 2856-2865 (2009)

13. Bai, L, Dai, B: Existence and multiplicity of solutions for an impulsive boundary value problem with a parameter via critical point theory. Math. Comput. Model. 53(9), 1844-1855 (2011)

14. Bai, L, Dai, B: Three solutions for a p-Laplacian boundary value problem with impulsive effects. Appl. Math. Comput. 217(24), 9895-9904 (2011)

15. Xie, J, Luo, Z: Subharmonic solutions with prescribed minimal period of an impulsive forced pendulum equation. Appl. Math. Lett. 52, 169-175 (2016)

16. Zeidler, E: Nonlinear Functional Analysis and Its Applications: Ill: Variational Methods and Optimization. Springer, Media (2013)

17. Rabinowitz, PH, et al.: Minimax Methods in Critical Point Theory with Applications to Differential Equations. Am. Math. Soc., Providence (1986)

\section{Submit your manuscript to a SpringerOpen ${ }^{\ominus}$ journal and benefit from:}

- Convenient online submission

Rigorous peer review

- Immediate publication on acceptance

- Open access: articles freely available online

- High visibility within the field

- Retaining the copyright to your article 